\title{
La teoría del derecho de contratos como desafío
}

\section{The Theory of Contract Law as a Challenge}

Esteban Pereira Fredes ${ }^{\star}$

Recepción: 05/11/2021

Evaluación: 25/11/2021

Aceptación final: 02/12/2021

Resumen: El presente trabajo tiene por objetivo mostrar el modo en que ha transitado la discusión acerca de la teoría del derecho contractual, tomando como punto de partida el diálogo que se desarrolla en el número de Discusiones titulado "Contratos: entre el derecho y la justicia”. Se da cuenta del debate esgrimido entre el autor y sus comentaristas y, enseguida, se añaden consideraciones críticas respecto de la teoría del derecho contractual ofrecida por Hevia. Por último, son apuntadas observaciones acerca del desarrollo y las posibles proyecciones de la reflexión filosófica sobre el derecho de contratos.

Palabras clave: contratos, derecho contractual, filosofía del derecho de contratos, teoría del derecho contractual.

Doctor en Derecho, Universidad de Girona. Profesor de Teoría del Derecho y Derecho Privado, Facultad de Derecho, Universidad Adolfo Ibáñez, Santiago de Chile. Correo electrónico: esteban.pereira@uai.cl. Agradezco a Diego Dei Vecchi por su generosa invitación para efectuar este balance y, en especial, por su entusiasmo y paciencia para que este trabajo llegara a buen puerto. Del mismo modo, a Diego M. Papayannis por impulsarme a desarrollarlo y al evaluador anónimo por sus observaciones y valiosas sugerencias. 


\begin{abstract}
The objective of this work is to show the way in which the discussion about the contract law theory has progressed, taking as a starting point the dialogue that takes place in the number of Discusiones entitled "Contracts: between law and justice". It gives an account of the debate wielded between the author and his commentators and, immediately, critical considerations regarding the contract law theory offered by Hevia are added. Finally, observations about the development and possible projections of the philosophical reflection on contract law are pointed out.
\end{abstract}

Keywords: contracts, contract law, philosophy of contract law, contract law theory.

\title{
1. Introducción
}

En Discusiones XV es desarrollado un fecundo diálogo acerca de cuestiones relativas a la justificación y los objetivos del derecho de contratos. Tal ejercicio es desplegado a partir del trabajo de Martín Hevia "Razonabilidad y responsabilidad: Rawls, Kant y la teoría del derecho contractual”, el cual es críticamente revisado por Mauricio Rengifo Gardeazábal, Iñigo de la Maza Gazmuri y Diego M. Papayannis.

En estas coordenadas, el objetivo del presente trabajo es mostrar la manera en que ha transitado la discusión esgrimida en ese número en el contexto de la filosofía del derecho de contratos. El trabajo está construido sobre la base de tres secciones. En la segunda sección, se resumen brevemente los planteamientos esgrimidos en el marco de aquel número. En la tercera sección, en tanto, se formulan algunas consideraciones sobre el debate acerca de la teoría del derecho contractual en diálogo con la teoría de Hevia. En la cuarta sección, por último, se devela el modo en que se ha desarrollado y cómo debería proseguir la reflexión filosófica sobre el derecho de contratos. 


\section{Contratos: entre el derecho y la justicia}

Un aspecto que de manera inmediata captura la atención de quien revise ese número es el título en virtud del cual se agrupan los trabajos allí contenidos. La relación entre el derecho y la justicia puede ciertamente instanciarse acudiendo a la figura contractual. Esto parece ser un rasgo común cuando se trata de nociones institucionales y no una peculiaridad exclusiva del derecho privado. Sin embargo, es efectivo que esta parcela de lo jurídico es generosa al momento de proveer de este tipo de nociones, tal como ocurre con el contrato, la propiedad, la responsabilidad, la familia o las sucesiones. Por ello, la articulación de una teoría del contrato debe atender tanto a consideraciones jurídicas como normativas. El tránsito entre el derecho y la justicia invita precisamente a la configuración de una teoría del derecho contractual que posea aptitud para explicar y justificar el contrato en las prácticas contractuales que tienen lugar del modo en que las conocemos.

La empresa de Hevia intenta conjugar ambas consideraciones para ofrecer una teoría del derecho de contratos con base en relaciones de interacción justas. Esta clase de relaciones, piensa el autor, deben entenderse formuladas a la luz de la idea de reciprocidad. Dicho criterio normativo exige que las condiciones y los términos de un vínculo contractual sean convenidas por los contratantes y que no sean fruto, por el contrario, de la imposición unilateral de una parte a la otra. De modo tal que, al tratarse de condiciones libremente acordadas por las partes de un contrato, es honrada la concepción de las personas como individuos libres e iguales para vincularse con las demás a partir de interacciones consentidas y justas. En este sentido, la reciprocidad ofrece en su esquema un criterio de corrección moral acerca de las relaciones contractuales: un contrato será justo en la medida en que esté basado en términos de interacción que hayan sido convenidos por las partes y, por el contrario, un acuerdo no será justo al defraudar las interacciones razonables mediante el establecimiento unilateral del contenido contractual. Del mismo modo, las reglas contractuales deben diseñarse en función de condiciones justas de interacción.

Estas observaciones tienen raíces intelectuales bien definidas. Se insertan en la tradición del contractualismo en filosofía política y de las éticas deontológicas en filosofía moral, echando mano de los enfoques de John 
Rawls e Immanuel Kant, respectivamente. Mientras que del primero se desprende el posicionamiento del derecho de contratos en la estructura social general, del segundo es extraída la teoría jurídica contractual en particular. Hevia parece suponer que tal complementación es necesaria porque el primero estaría más bien preocupado por el derecho público que en el privado. Por ello, Kant viene a colmar este vacío a partir de un prisma sensible a los acuerdos entre particulares. Para dar cuenta de la estructura general de las instituciones sociales justas es menester atender a Rawls y, en tanto, si el objetivo es la formulación de interacciones privadas justas es indispensable acudir al pensamiento kantiano ${ }^{1}$.

La teoría del derecho contractual que el autor propone está comprometida con la división de la responsabilidad rawlsiana y, a su vez, con las ideas de persona razonable y deberes relacionales. Como es sabido, Rawls configuró su monumental teoría de la justicia en aras de una 'sociedad bien ordenada' en la cual existe una concepción pública de la justicia, el diseño de las instituciones se ajusta con tales principios públicos de justicia y estos, a su vez, son discernidos y puestos en práctica por los ciudadanos (Rawls, 1995b, pp. 18-19; 2004, pp. 31 y ss.). En este marco teórico está posicionada la división de la responsabilidad según la cual esta idea se encuentra escindida en dos dimensiones. En primer lugar, se encuentra la dimensión pública de la responsabilidad en la cual tienen lugar ciertos deberes no relacionales relativos al apoyo de instituciones sociales justas y que no se deben respecto de personas en concreto. En segundo lugar, en tanto, está el ámbito que -con ciertas reservas- podría entenderse como la dimensión privada de la responsabilidad en que se presentan los deberes relacionales, es decir, demandas entre las personas. Estas últimas pueden perseguir forjar sus planes personales de vida en la medida en que también

1 Resulta interesante observar que esta estrategia es afín con la implementada por Ernest J. Weinrib sobre la justicia correctiva en el derecho privado. De acuerdo con esta, es necesario combinar la visión aristotélica con la kantiana, dado que el primero proporciona la estructura formal de la justicia correctiva y el segundo, por su parte, brinda una dimensión normativa centrada en los derechos de autodeterminación de los agentes libres. De nuevo, la justificación de esta conexión reside en que un modelo efectúa un trabajo que el anterior no realiza. Sobre esta cuestión, véase, Weinrib, 2017, en particular, pp. 117-144. También esto se destaca en Weinrib (2012, pp. 9-37). 
permitan a las demás alcanzar sus proyectos de vida, exigiendo a los individuos responsabilizarse por sus propias vidas. Ambas dimensiones encarnan una concepción normativa de la persona según la cual en esta se conjuga su carácter de agente racional y razonable. De ahí que los términos justos de la interacción entre las personas apelen a estos rasgos en un espacio de reconocimiento público y reciprocidad para con las demás (Rawls, 2004, p. 29). ${ }^{2}$ No es posible, entonces, que una persona se vincule con otra sin haber consentido a hacerlo y las reglas del derecho contractual deben establecer los límites recíprocos para que se garantice la libertad de cada individuo.

El pensamiento jurídico de Kant, por su parte, se encarga de proporcionar una genuina teoría del derecho privado, según es entendido por Hevia. Por ende, el esquema anterior se encaja con el modelo kantiano toda vez que hay una continuidad teórica entre ambas propuestas. Así, la capacidad que tienen las personas de desarrollar una concepción de lo bueno tiene su correlato en la noción de independencia de la teoría jurídica kantiana. Tal noción alude a la aptitud que tienen las personas, a diferencia de las cosas, de definir sus planes de actuación y elegir los medios más idóneos para alcanzarlos. Esta capacidad importa que cada persona puede determinar sus planes vitales de manera independiente de los demás individuos. La búsqueda de los planes personales de cada persona requiere la existencia de límites recíprocos a la libertad en el marco de los diferentes modos en que interactúan los individuos. Desde el prisma de Kant, entonces, es posible restringir la libertad para protegerla y de ahí que una privación del derecho que detentan las personas de usar los medios de la manera en que estimen conveniente autoriza la limitación de su libertad (Kant, 1999, 231§C, p. 39). No se encuentra permitido violar el derecho de las demás personas a usar los medios según lo dispongan, sin que medie su consentimiento, aun cuando tal transgresión se efectúe a propósito de la consecución de un proyecto personal.

2 De acuerdo con Rawls, "[l]os términos justos de la cooperación articulan la idea de reciprocidad y mutualidad; todos los que cooperan deben salir beneficiados y compartir las cargas comunes, de la manera como se juzga según un punto de comparación apropiado" (Rawls, 1995a, p. 279). 
Estas consideraciones arriban a una teoría del derecho contractual articulada sobre la base del consentimiento de los contratantes en el acuerdo y este, en particular, posee un carácter público asociado a las exigencias de tratar a las demás personas en tanto iguales. Por ello, el estándar apropiado para la configuración de la relación contractual supone un comportamiento justo en atención a su interacción con los demás. Este estándar se encuentra en sintonía con la concepción normativa de persona razonable que ocupa un lugar central en las relaciones de derecho privado. Su visión objetiva se extiende a la del consentimiento, que también es entendido mediante un enfoque objetivo. La trascendencia del consentimiento explica por qué el establecimiento de los términos del contrato de manera unilateral por una de las partes deviene irrazonable, pues deja a un contratante a merced del otro. La apuesta de Hevia es demandar a las personas un comportamiento en interacción con otros y no uno que atienda a lo que particularmente consideran como justo. El consentimiento, por tanto, se interpreta en términos de un estándar público y asegura que las personas iguales se relacionen en condiciones justas de interacción. ${ }^{3}$

Este despliegue argumentativo es puesto a prueba por Rengifo, De la Maza y Papayannis. Sus comentarios críticos pueden agruparse en atención al foco en que se concentra cada autor. Mientras que los dos primeros analizan la propuesta desde su falta de encaje con instituciones y figuras de las prácticas contractuales, el último la tematiza a partir de sus presupuestos e incidencias. Rengifo apunta a que la teoría de Hevia responde a una imagen de la institución contractual que no es consistente con la posición preeminente que ocupa la libertad en la tesis propuesta. Echa mano a las condiciones generales de contratación y a las situaciones contractuales de hecho como reflejos de la alteración en el modo tradicional de entender el contrato y conspicuas limitaciones a la libertad contractual de las partes, develando la incompatibilidad que media entre esas figuras y la visión tradicional del contrato (Rengifo, 2014, pp. 83-84). Por ello, la comprensión de Hevia parece más bien propia de otra realidad contractual y no resulta

3 El trabajo analizado constituye una selección de la acabada obra que se encuentra en Hevia (2013). Esta continúa siendo, por supuesto, la obra más relevante en filosofía del derecho de contratos surgida en la comunidad académica del ámbito iberoamericano. 
pertinente en los contextos actuales de contratación en que la libertad se encuentra fuertemente debilitada. De la Maza, por su parte, continúa esta ruta acerca de la falta de adecuación de la teoría de Hevia con la figura del contrato de adhesión, el objeto del contrato, las acciones frente al incumplimiento de lo convenido. A diferencia del planteamiento de Rengifo en el cual hay una evaluación crítica al empleo de la filosofía kantiana para nutrir su teoría acerca del derecho contractual, De la Maza clarifica que no se ocupará del tratamiento efectuado sobre Rawls ni su conciliación con Kant, indicando que su prisma de análisis proviene, en realidad, de la dogmática civil.

Conviene detenerse en el problema que generan los contratos de adhesión a la idea de reciprocidad y la conformación de reglas contractuales que garanticen intercambios justos. Esta clase de vínculos contractuales se caracteriza precisamente porque los términos han sido establecidos unilateralmente por una de las partes a la otra, y son frecuentemente impuestos por el contratante que está posicionado en una condición favorable y aventajada en términos económicos. Hevia parece estar aliado con la célebre frase de Fouillée según "quien dice contractual dice justo", pero las relaciones de consumo están asociadas a la idea inversa, esto es, "quien dice justo dice contractual". La justicia de la interacción que tiene lugar en el contrato de adhesión no está asociada, en verdad, al contenido del acuerdo, sino que con aquello que se encuentra fuera de este (De la Maza, 2014 , p. 90). Si se nota el significativo impacto que tienen los contratos de adhesión y las relaciones de consumo en la contratación contemporánea, la justicia de la interacción no puede únicamente juzgarse a la luz de la unilateralidad de lo convenido, que atenta con la idea de reciprocidad y el consentimiento contractual.

Papayannis, por último, sitúa su examen crítico tanto en los presupuestos como en la incidencia de la teoría del derecho contractual de Hevia. A nivel de presupuestos, Papayannis posiciona correctamente la perspectiva de Hevia en el marco de la línea de reflexiones característica de la escuela de Toronto en filosofía del derecho privado. Gracias a la comunión del autor con este influyente prisma, extrae dos asunciones altamente problemáticas. De un lado, el énfasis en la bilateralidad de las relaciones de derecho privado. De otro, la comprensión y ordenación de cuestiones cen- 
trales de esta parcela de lo jurídico como la celebración y el cumplimiento de los contratos, así como la compensación de los daños ocasionados en nuestros encuentros espontáneos, a la luz de la justicia correctiva. Al entrelazar ambas consideraciones se advierte que la estructura bilateral de la relación de derecho privado únicamente contiene razones que son relativas a los agentes y no son neutrales a estos. Tal observación debe articularse en términos unitarios y exclusivos. El derecho privado en su generalidad -y especialmente el derecho de contratos- encarna el vínculo bilateral existente entre las partes y ajusta íntegramente sus interacciones a la justicia correctiva. Desde luego, el espacio que queda al margen es aquel que podría ocupar la justicia distributiva en el derecho privado.

Desde el punto de vista de Papayannis, ambas asunciones son erróneas del modo en que son entendidas en el esquema de Hevia y ofrecen dificultades para la comprensión del derecho privado. En relación con la estructura bilateral, el autor acude a un conjunto de consideraciones que progresivamente han ganado terreno en el derecho de contratos de la tradición jurídica continental. Estas versan sobre las excepciones que hoy en día presentan los principios fundamentales de la contratación como la autonomía de la voluntad, el consensualismo y, en especial, el efecto relativo de los contratos. Dichas excepciones no solo tensionan la incuestionable vigencia de la bilateralidad, sino que también ponen de relieve el hecho de que la relación de derecho privado no se funda uniformemente en cánones de justicia correctiva. Al respecto, Papayannis aborda el compromiso del derecho privado con la justicia correctiva a partir del derecho contractual. Allí muestra que el principio de responsabilidad sobre la base del cual Hevia esgrime su teoría se fundamenta, en verdad, en consideraciones de justicia distributiva. Del mismo modo, los derechos y deberes que emanan para los contratantes una vez que estos celebran el acuerdo no provienen de la autonomía de la voluntad y el consentimiento, en tanto, opera en el momento de la constitución de la relación contractual, pero de modo alguno resulta exhaustivo sobre las regulaciones que guían esas interacciones. La fortaleza con que la escuela de Toronto interpreta la justicia correctiva es desplazada por la insoportable levedad que esta experimenta para dar cuenta de los distintos ámbitos del derecho privado, lo cual contribuye a echar luz sobre la dimensión pública del derecho privado (Papayannis, 2014, p. 143). En efecto, la repercusión de adoptar las directrices de Hevia 
está reflejada, por una parte, en el hecho de que se resiente la posibilidad de efectuar una teoría del derecho contractual y, por otra, en que el rasgo privado del derecho privado oculta su innegable carácter público.

En la próxima sección de este trabajo se introducirán algunas consideraciones sobre la teoría del derecho de contratos que pueden ensanchar los órdenes de análisis de la propuesta teórica brindada por Hevia.

\section{Hevia y la teoría del derecho contractual}

Los comentarios críticos antes reseñados resultaron - como se pudiere haber anticipado- estimulantes para el autor y ello es patente en las respuestas que formula para cerrar la valiosa discusión que contiene el número (Hevia, 2014b, pp. 145-168). Quisiere continuar con este ejercicio y añadir otros ángulos gracias a los cuales es posible persistir en la reflexión sobre la teoría del derecho de contratos presentada por Hevia. Hay tres puntos sobre los cuales conviene detenerse.

En primer lugar, la teoría de Hevia se encuentra construida con base en los planteamientos de Rawls y Kant. A pesar del agudo y sofisticado empalme que logra el autor respecto de ambas posiciones, resulta interesante evidenciar las razones que abonan esta conciliación. Según Hevia, la necesidad de sumar la teoría kantiana del derecho contractual al esquema teórico provisto por Rawls reside en que este último estaba preocupado del derecho público y no del derecho privado. De ahí que naturalmente su marco teórico carezca de una teoría del derecho de contratos. Sin embargo, la omisión en que habría incurrido la teoría de la justicia rawlsiana del derecho privado es una cuestión que no está del todo clara, pero que para Hevia constituye un presupuesto inicial de su trabajo. La literatura se ha mostrado atenta a esta consideración y buena parte de los estudios coinciden en que la teoría de la justicia de Rawls no puede pasar absolutamente por alto el rol del derecho privado en la configuración de una sociedad bien ordenada, o bien, si se quiere, que el derecho privado es perfectamente encajable en la estructura general de las instituciones sociales que articula Rawls (Rawls, 1995b, p. 155). El tratamiento que este último ofrece en su obra es antes un problema de énfasis que de efectiva omisión. 
Este constituye un asunto relevante para la tesis de Hevia porque en sus términos Rawls obvia tanto el derecho privado como el derecho de contratos. No obstante, hay esfuerzos tendientes a mostrar cómo podría contribuir el diseño de un derecho contractual justo en una sociedad que podamos calificar de justa (Scheffler, 2015, pp. 213-235). Tal esfuerzo es precisamente alentado a partir de institutos del derecho de contratos que auxilian desde el derecho privado la empresa rawlsiana. ${ }^{4}$ Como se podrá observar, el tratamiento que esgrime Hevia acerca de ambas líneas de pensamiento es asimétrico tanto en su reconstrucción como en la incidencia que aquellas presentan en su tesis final. Resulta evidente que la teoría de Rawls se encuentra más desarrollada que la de Kant, en circunstancias que el primero carece de aquello que sí posee el segundo, esto es, derecho contractual. Del mismo modo, es el marco teórico de Rawls el que efectivamente delinea las ideas de división de responsabilidad, interacciones justas, reciprocidad y consentimiento que son cruciales para la teoría del derecho contractual de Hevia. Cabe cuestionarse, entonces, cuál es el verdadero rendimiento epistémico de la teoría kantiana en la propuesta del autor. El compromiso por la autonomía de la voluntad y la conformación de relaciones entre personas libres e iguales se encuentra fuertemente defendida en el esquema rawlsiano y, en verdad, se trata de la posición de mayoría en la filosofía moral y política de corte liberal. ¿Cuál sería, en definitiva, la pérdida sustantiva al interpretar la comprensión del derecho contractual desarrollada por Hevia exclusivamente en términos rawlsianos? Quizá ello serviría para revelar que tal núcleo de reflexiones es efectivamente pertinente para albergar una teoría del derecho de contratos. ${ }^{5}$

En segundo lugar, una cuestión pendiente es la calificación de la teoría presentada por Hevia de acuerdo con su compromiso con un valor unitario o bien por un conjunto variado de ellos. Su tesis admite ser interpretada

4 A partir de la institución de la ventaja injusta que proscribe el aprovechamiento de una parte contratante a la otra ha sido tematizada la incidencia del derecho de contratos en la verificación de las condiciones de la justicia de corte rawlsiana. Desde estas coordenadas, véase Barceló Compte (2019, en especial, pp. 33-42). Sobre el lugar que ocupa la justicia distributiva en la empresa rawlsiana, véase Freeman (2018).

5 Sobre este tipo de consideraciones puede resultar especialmente iluminadora la idea de la base pública de la justificación del contrato por la cual aboga Peter Benson (1995, pp. 
como una teoría monista entendiendo que lo valioso de la relación contractual y del derecho de contratos reside en el consentimiento alcanzado por personas libres e iguales y que garantiza la reciprocidad de la interacción entre los contratantes. Dicha lectura esgrime la operatividad de un criterio normativo común para la diversidad de relaciones contractuales que tienen lugar. En efecto, la cuestión acerca de qué cuenta como una interacción realizada en condiciones justas debe ser resuelta con cargo a la existencia del consentimiento de las partes que genera el acuerdo y que, por el contrario, este no provenga de la imposición unilateral de los términos de un contratante al otro. Tal criterio puede ser válido para una parte de la contratación, pero enfrenta severas dificultades para dar cuenta de la generalidad de las prácticas contractuales en que el consentimiento no juega el rol preponderante que Hevia le reconoce, ni tampoco la interacción se forja entre personas libres e iguales. ${ }^{6}$ Como fue visto a propósito del contrato por adhesión y las relaciones de consumo, las condiciones son establecidas unilateralmente por uno de los contratantes quien se encuentra, a su vez, en una posición contractual en que los parámetros de libertad e igualdad están profundamente desafiados. Allí podría considerarse el criterio formulado por Hevia como insuficiente, toda vez que no podría desatender la manera en que se desarrolla, de hecho, la contratación y que no encaja con la descripción que ofrece la teoría. ${ }^{7}$

Por supuesto, la teoría de Hevia podría reinterpretarse como una de corte pluralista, es decir, que reconoce la presencia de una diversidad de valores o criterios para determinar cuándo y bajo qué términos una relación contractual corresponde a una interacción justa que debe ampararse por el derecho de contratos. Pero tal ejercicio supone explicitar cuáles serían estos otros valores o criterios normativos. De la manera en que es presentada la teoría, esta no podría ser calificada como dualista ni mucho menos como pluralista. Como sea que fuere el caso, la introducción de

273-336). Su incidencia para la construcción de una teoría del derecho contractual está en Benson (2019, en particular, pp. 1-31).

6 Algunos de los límites con que se enfrenta el consentimiento en el derecho de contratos se encuentran analizados en Hevia (2018, pp. 107-118).

7 Acerca de las variaciones que ha sufrido el paradigma clásico del contrato y rol moderno que juega el derecho contractual, véase Atiyah (1986b, pp. 1-9). 
otros valores o criterios de corrección adicionales para las interacciones acarrea otros retos que están asociados a la clarificación del estatus que media entre esos valores. En otros términos, sería necesario especificar si alguno de estos tiene una posición preeminente sobre otros o bien si están situados en lugares equivalentes. El modo en que estos se relacionan entre sí, y cómo estos deben ser implementados, constituye un asunto de primera importancia frente a un escenario en que las condiciones de contratación han variado sustantivamente. La dificultad que se aprecia en la teoría de Hevia y su compromiso monista es que esta se encuentra formulada sobre la base de una visión del contrato que no tiene un encaje cómodo con la realidad contractual. Se trata más bien de una concepción del contrato que no responde a las modalidades en que hoy en día entendemos las prácticas contractuales, mostrándose deudora de la vieja ortodoxia del derecho de contratos (Scheinman, 2000, p. 206). La apuesta monista de Hevia tampoco da en el clavo sobre el tipo de contrato que encarna el derecho contractual.

Una cuestión adicional sobre este punto radica en que la filosofía del derecho de contratos ha problematizado los caracteres de las personas que participan en la relación contractual, repensando los estándares de libertad e igualdad en el derecho de contratos y, si esto es correcto, en el derecho privado. Para Hevia las relaciones contractuales en términos de interacciones justas tienen precisamente lugar entre personas libres e iguales. Pese a que en su trabajo no hay rastros relativos a cómo deben entenderse ambas nociones, resulta evidente de que se trata de estándares normativos que la relación contractual debe satisfacer. Si bien el derecho privado, desde su configuración moderna en el ámbito jurídico continental establecida gracias a la codificación, adoptó una aproximación meramente formal acerca de las personas como sujetos de relaciones jurídicas privadas, paulatinamente tales rasgos han avanzado a dimensiones sustantivas o materiales. ${ }^{8}$ Como un modo de simplificación de los destinatarios de las reglas jurídicas, los agentes fueron formalmente entendidos como libres e iguales, estableciendo un sujeto único de las relaciones de derecho (Tarello, 2002, pp. 55-59). Esto desplaza la pertinencia de consideraciones particulares

8 En contra de la lectura absolutista e individualista que suele imputarse al Code de $1804 \mathrm{y}$ que se propagó al derecho privado moderno fundado en la tradición continental, véase Gordley (1994, pp. 459-505). 
a favor de algunas personas respecto de las cuales puede cuestionarse su real libertad para participar en interacciones de derecho privado o la posición paritaria en que efectivamente se encuentran para desplegarlas. ${ }^{9}$ Tanto la noción de libertad como la de igualdad han sido objeto de una revisión en términos de rescatar su sustancialidad o materialidad, abandonando el prisma meramente formal con que se les suele analizar en derecho privado. Lo interesante radica en que quienes han abogado por esta clase de empresa lo han hecho precisamente para fundar en tales nociones relaciones justas y no solo relaciones de derecho privado. ${ }^{10}$ Del mismo modo, Hevia procura alcanzar este tipo de relaciones a través de las condiciones de interacción justas, mas lo intenta sin efectuar la relectura de la libertad y de la igualdad. ${ }^{11}$

Aquí surge de nuevo la dificultad a la que debe hacer frente la teoría de Hevia al ofrecer un esquema unitario y compartido sobre el derecho contractual a partir de una comprensión tradicional del contrato. El monismo de su empresa echa mano a una visión de la institución contractual que muestra rigidez para adaptarse a las distintas dimensiones y variantes que caracterizan el contrato en las prácticas sociales, tal como cotidianamente lo conocemos y participamos de este. Dicha concepción del vínculo contractual está forjada sobre la base de las ideas de libertad e igualdad de las partes, el consentimiento y la bilateralidad de la relación de derecho privado. Desde luego, esta forma de entender el contrato se encuentra más asociada al esquema decimonónico de contratación que al actual y de ahí que los cargos contra la propuesta de Hevia sobre el espectro del fenómeno contractual que su teoría desatiende o no logra explicar resultan ciertamente apropiados. No obstante, el tránsito desde esa comprensión particular sobre el contrato a la construcción de una teoría del derecho contractual supone cubrir no solo la generalidad del fenómeno sino también dar cuenta del tipo de fenómeno que es el contractual. Desde mi punto de

9 Del mismo modo, las consideraciones unilaterales esgrimidas a favor de una parte de la relación de derecho privado han sido consideradas como inconsistentes con la igualdad transaccional y bilateralidad de la justicia correctiva. Véase, por ejemplo, Weinrib (2017, p. 17).

${ }^{10}$ Este reclamo se encuentra formulado en (Dagan y Dorfman, 2016, pp. 1395-1460). Para una evaluación crítica de dicha perspectiva, véase Gardner (2017, pp. 179-201).

${ }^{11}$ Un esfuerzo de reinterpretación de estas nociones en la teoría del derecho contractual está disponible en Dagan y Heller (2017, pp. 41-47 y pp. 67-92). 
vista, el derecho de contratos tiene como uno de sus rasgos distintivos la complejidad que se manifiesta en la estructura, composición y los compromisos normativos de esta parcela de lo jurídico. El marco teórico mediante el cual se busca explicar y justificar el fenómeno debe capturar sus características y el modo en que este se desarrolla. Por ello, todo esquema que opte por resguardar y honrar la supuesta uniformidad del derecho contractual presentará dificultades para desarrollar su tarea porque sencillamente este no es el marco adecuado para abordar el fenómeno contractual.

En tercer lugar, por último, es relevante tematizar la manera en que Hevia, siguiendo la taxonomía de Stephen A. Smith, califica su visión del derecho contractual como una teoría interpretativa (Smith, 2004, p. 4). Este tipo de teorías acerca del derecho de contratos "tienen como objetivo mejorar la comprensión del derecho, poniendo de relieve su importancia o significado" (Smith, 2004, p. 5). De este modo, se debería entender que la teoría del derecho contractual de Hevia busca brindar una explicación de este ámbito que resalta su relevancia o valor. La formulación de términos de interacción justos que aseguren el respeto por el consentimiento y la reciprocidad de las partes, evitando el establecimiento unilateral del contenido de los acuerdos, muestra la importancia del derecho de contratos al refinar la manera en que nos relacionamos mutuamente y el valor de forjar vínculos contractuales entre personas libres e iguales. Desde luego, esta versión revela un significado valioso que posee el derecho de contratos y lo ubica en un lugar preponderante del fenómeno jurídico. En estos términos, la perspectiva de Hevia es efectivamente una teoría interpretativa.

Si bien tal calificación es apropiada, no deja de llamar la atención que el despliegue argumentativo del autor desatienda el papel de las instituciones que forman parte de esta parcela de lo jurídico, con el objeto de echar luz sobre su importancia y significado en las prácticas sociales. Una estrategia que resulta especialmente pertinente con la teoría interpretativa sobre el derecho de contratos es mostrar dichas dimensiones valiosas a partir de algunos institutos que lo conforman. ${ }^{12}$ El tratamiento que esgrime Hevia, en cambio, prescinde de este prisma y solo se concentra en la institución

12 Tal esquema se encuentra implementado para efectos de desentrañar los componentes altruistas que yacen en la estructura normativa del derecho de contratos en Pereira Fredes, 
contractual. Tal consideración es relevante en la medida en que una lectura institucional de lo jurídico resulta particularmente valiosa para agudizar nuestra comprensión del derecho contractual. Dicha perspectiva teórica subraya las dimensiones normativas o valorativas del derecho y articula su tarea sacando partido a instituciones distintivas del área de la cual se trate (MacCormick, 2011, pp. 27-58). ¿Por qué, entonces, elaborar una teoría interpretativa acerca del derecho de contratos y obviar sus institutos característicos? La concepción tradicional del contrato que subyace a la teoría de Hevia puede precisamente ponerse a prueba acudiendo a instituciones representativas del derecho contractual que hoy en día conocemos, como ocurre con la teoría de la imprevisión, la lesión subjetiva-objetiva, la ventaja injusta, el abuso del derecho, entre otras. Gracias a estas es posible desafiar la imagen paritaria y bilateral del contrato, así como los contornos exclusivamente delineados por el consentimiento de los contratantes. ${ }^{13}$

Un instituto que se echa especialmente en falta es la buena fe. De manera progresiva la buena fe ha cobrado una relevancia crucial para la reflexión dogmática y filosófica de las relaciones contractuales y el derecho de contratos. La cuestión acerca de cómo la perspectiva de Hevia puede engarzarse con el funcionamiento y las diferentes demandas de la buena fe resulta determinante porque al menos en el contexto jurídico continental, con cargo a la buena fe, no solo importa el texto sino el subtexto de la relación contractual. ${ }^{14}$ Asimismo, sus deberes no están únicamente orientados a no dañar los intereses de la otra parte del contrato, sino también en ocasiones a beneficiarlos, instando a un contratante a actuar positivamente a favor de aquellos. Esto revela que en instituciones como la buena fe se observa que las nociones de consentimiento y reciprocidad, del modo en

2020a (en especial, pp. 255 y ss.).

${ }^{13}$ De igual manera, a partir de ciertos institutos puede tematizarse el real compromiso del derecho de contratos por la justicia de nuestros acuerdos, poniendo de relieve que este ámbito no solo está preocupado de la justicia procedimental de los intercambios sino también de la justicia sustantiva o del resultado de los contratos. Al respecto, véase Atiyah, 1986a (pp. 329-354).

14 Desde estas coordenadas, puede anotarse, por ejemplo, el impacto de la teoría relacional del contrato en la extensión de los deberes contractuales de las partes. Sobre este enfoque, véase Macneil, 1974, pp. 691-816. 
que Hevia las entiende, deben ser repensadas. El contrato no solo obliga a lo que las partes expresa y consentidamente han convenido y, a su vez, la actuación de buena fe no solo importa para el contratante honrar un estándar de reciprocidad relativo a la abstención de lesionar a la otra parte, pues en ciertas situaciones se le exige derechamente favorecer sus intereses contractuales. ${ }^{15}$

Esta posible aproximación institucional permitiría, por una parte, reforzar la conexión entre la teoría del derecho contractual de Hevia y sus raíces rawlsianas y, por otra, engarzar su visión interpretativa con las coordenadas dworkinianas. En relación con la primera arista, la atención de Rawls sobre las instituciones sociales invita a tomar en cuenta a institutos como el contractual. Como antes fue sugerido, resulta indispensable determinar el papel que cumple el diseño del derecho de contratos para conformar sociedades justas. ${ }^{16}$ A pesar del influjo que reconoce Hevia de la obra de Rawls en su planteamiento, esta no se ve, desde mi modo de ver las cosas, genuinamente reflejada en el análisis de instituciones del derecho de contratos. De esta manera, su examen pudiere mostrar en qué sentido ciertas instituciones del derecho contractual encarnan y fomentan los ideales rawlsianos para forjar relaciones entre contratantes considerados como libres e iguales. A partir de ello, la cuestión sobre el rol de la estructura del derecho contractual en la estructura general de las instituciones sociales

${ }^{15}$ De ahí que la composición normativa de la buena fe no es uniforme, sino que está formulada en términos bifrontes y, por ello, resulta indispensable acudir a modelos de fundamentación normativa que atiendan a ambas direcciones. De este modo, se ha propuesto que la buena fe replica un rasgo central del derecho de contratos; a saber, que hay una convivencia de parámetros del individualismo desinteresado y del altruismo moderado. Acerca de esta interpretación y su rol para capturar la complejidad del derecho contractual, véase Pereira Fredes, 2020b (pp. 111-142).

${ }^{16} \mathrm{Al}$ respecto, Samuel Scheffler ha advertido que "la idea de que el derecho privado en su conjunto se encuentra excluido de la estructura básica es claramente insostenible, ya que 'las formas legalmente reconocidas de propiedad' se incluyen repetidamente entre los ejemplos canónicos de Rawls sobre instituciones que pertenecen a la estructura básica. Este explícitamente sitúa el derecho de propiedad dentro de la estructura básica, donde ocupa su lugar junto a cosas tales como 'los mercados competitivos' y 'la estructura de la economía. Y esto dificulta ver cómo el derecho de propiedad, los mercados competitivos y la estructura de la economía podrían formar parte de la estructura básica, mientras que el derecho contractual no" (Scheffler, 2015, p. 219). 
sería inevitable. Sin duda, tal ejercicio estrecharía su ligazón con Rawls, pero además evitaría los obstáculos que parece enfrentar una teoría interpretativa sin instituciones.

Respecto de la segunda cuestión, en tanto, puede que no constituya un anhelo del autor emparentar su posición con la de Dworkin, pero, de nuevo, el carácter interpretativo de su teoría podría resultar robustecido si es que ello importa, como lo piensa Smith, echar luz sobre la importancia o significado del derecho contractual. Este rasgo de las visiones interpretativas del derecho de contratos encuentra en el esquema dworkiniano un aliado importante, ya que refleja la fuerte incidencia del ámbito institucional para efectos de mostrar las prácticas jurídicas en su mejor luz, develando el sentido de estas y su valor distintivo (Dworkin, 2012b, pp. 159-228). El derecho de contratos, en efecto, se desenvuelve en un cúmulo de prácticas sociales que debieren significar algo para quienes en ellas participan. No debemos olvidar que el modelo teórico de Hevia es marcadamente normativo y de ahí que las consideraciones valorativas sobre el derecho contractual son inevitables. Así, la interpretación constructiva se encuentra al servicio de ofrecer la mejor justificación de la práctica, procurando convertirla en lo que mejor que puede llegar a ser (Dworkin, 2012a, p. 11). Después de todo, y como insiste el título de este número, se trata de posicionar el contrato, entre el derecho y la justicia. No debería entonces abstraerse de esta empresa a la propia teoría con la cual se interpreta el derecho de contratos.

En la sección final de este trabajo se apuntarán unas notas sobre el modo en que ha transitado la reflexión filosófica acerca del derecho contractual, junto con algunas de sus posibles proyecciones.

\section{La senda de la filosofía del derecho de contratos}

La filosofía del derecho de contratos se ha desarrollado contemporáneamente bajo el alero de la filosofía del derecho privado y, por tanto, la manera en que se entiende esta última es decisiva para dar cuenta de la primera. El esfuerzo central de la filosofía del derecho privado ha estado puesto en determinar la identidad de este ámbito jurídico, así como en desentrañar sus fundamen- 
tos y propósitos representativos (Pereira Fredes, 2017; Papayannis y Pereira Fredes, 2018b). ${ }^{17}$ De este modo, la empresa teórica ha recorrido las diversas preocupaciones del derecho privado, tales como derecho de daños, derecho de propiedad, derecho de restituciones o enriquecimiento injustificado y, por supuesto, derecho de contratos. Esta sistematización entre el análisis filosófico acerca de los fundamentos y los propósitos del derecho contractual contribuye a mostrar cómo las investigaciones sobre esta área se han desarrollado. Tal observación revela que la discusión esgrimida por Hevia y sus comentaristas permanece absolutamente presente en la literatura, pero esta se ha especificado en las directrices trazadas sobre la base de lo que recientemente entendemos por filosofía del derecho privado.

El problema de la fundamentación normativa del derecho de contratos ha capturado la atención de esta línea de reflexiones. En el contexto contractual pueden pensarse en al menos dos cuestiones centrales. De una parte, la determinación de la justificación de la fuerza vinculante del contrato. De otra, el análisis filosófico de la fundamentación normativa del derecho contractual. Mientras que el primer asunto versa sobre la formulación del criterio valorativo que justifica la obligatoriedad del vínculo contractual, el segundo corresponde a una indagación acerca del fundamento normativo del derecho de contratos en general. Como puede observarse, ninguna de estas preocupaciones se encuentra esgrimida en la teoría del derecho contractual de Hevia ni son puestas de relieve por quienes la discuten. Por supuesto, una teoría del derecho contractual no puede prescindir de estos órdenes de análisis y hay buenas razones para sostener que con menor razón una teoría interpretativa del derecho de contratos podría desprenderse de ambos problemas.

Es cierto que la justificación normativa del efecto vinculante del contrato se alzó tempranamente como una de las principales preocupaciones de la filosofía del derecho de contratos y, con posterioridad, esta parece haber extraviado su nitidez en el escenario contractual. No obstante, esta cuestión merece ser revisitada y, en especial, cuando el foco de atención de la propuesta son las interacciones justas forjadas sobre la base del consentimiento de los contratantes. El consentimiento ha ocupado permanen-

17 Sobre el debate metodológico en la filosofía del derecho privado, véase Papayannis (2016b, pp. 439-448). 
temente un lugar predominante en la teoría general del contrato, siendo posicionado en el marco de los principios fundamentales de la contratación. Una cuestión significativa es que los estudios dogmáticos de derecho contractual otorgan indistintamente dos funciones al consentimiento de las partes, entendido en términos de un acuerdo entre sus voluntades. De un lado, opera como un requisito indispensable para la existencia del contrato $\mathrm{y}$, de otro, sirve de fundamento para el pacta sunt servanda del contrato. Esta operatividad dual es, a mi juicio, un yerro persistente. La voluntad o el consentimiento actúa a propósito de la formación de la relación contractual y no, en verdad, para justificar que las prestaciones deben cumplirse de manera incondicionada por las partes. La teoría del derecho de contratos que ofrece Hevia, como fue indicado, concentra su atención en el consentimiento para asegurar la reciprocidad de la relación contractual, mas no añade observaciones acerca de su potencial rol justificativo de la fuerza vinculante del contrato. En este sentido, es plausible asumir que en su teoría el criterio de justificación de la obligatoriedad del acuerdo reside en el respeto por el consentimiento o la voluntad de las partes. Si esto fuere así, la propuesta estaría nuevamente en aprietos porque tal criterio normativo no juega el rol que la visión tradicional del contrato le atribuyó. En buena parte de la contratación contemporánea el consentimiento se encuentra severamente debilitado $y$, en ocasiones, es urgente repensar su real vigencia en ciertas figuras contractuales. ${ }^{18}$

Más allá de esta posible dificultad, en la filosofía del derecho de contratos el consentimiento o la voluntad de los contratantes fue reemplazado por la fuerza apelativa de las promesas. La tesis canónica en esta línea de pensamientos es el modelo de Charles Fried, quien entiende que los contratos constituyen promesas y, por ende, obligan a las partes de la misma forma. Su estrategia fue conjugar una tesis conceptual y otra normativa. Así, dado que los contratos son promesas, los primeros obligan a los contratantes con la fuerza con que las segundas vinculan a quien las formula (Fried, 1996, pp. 19-44). Esta lectura ha generado una fecunda discusión

${ }^{18}$ Un examen sobre la idea de muerte del contrato en que se muestra en qué sentido no es posible descartar del todo que este - según una determinada versión- haya efectivamente muerto, a la luz de la falta de encaje de los postulados de la comprensión tradicional del 
sobre la pertinencia o no de asimilar ambas nociones (Papayannis y Pereira Fredes, 2018a, en especial, pp. 16-23). Uno de los motivos para la negativa es que la asimilación entre contrato y promesa importa la simplificación de la función en las relaciones sociales del contrato, toda vez que este contribuye a incrementar la autonomía de las partes, evitando forjar vínculos personales entre estas, con base en el propio instrumento contractual. El contrato es entendido como una opción frente a la promesa, porque en lugar de estrechar el vínculo personal, permite el distanciamiento entre los contratantes (Kimel, 2018, p. 176). La cuestión que me interesa resaltar es que la discusión acerca de la justificación de la fuerza vinculante del contrato ha girado a la investigación sobre las funciones distintivas del derecho de contratos. Por ello, no resulta baladí que el proyecto de Fried haya sido originalmente formulado - quizá de un modo optimista - como una teoría de la obligación contractual. A partir de la identidad entre el contrato y la promesa, entonces, podría construirse y sistematizarse una teoría general de lo contractual.

De ahí que otras alternativas han reorientado su interés en el respeto por la autonomía personal del contratante como un sustento para la obligatoriedad de lo pactado. ${ }^{19}$ Tal criterio tendría la ventaja de adaptarse de mejor manera al contexto actual de la contratación en que la participación de la voluntad de las partes se ha resentido. Del mismo modo, el respeto por la autonomía personal exige el estándar de bilateralidad en la medida en que un contratante debe observar lo que autónomamente ha acordado, pero también esa demanda supone honrar la actuación autónoma de la otra parte del contrato. A pesar de que el cambio de protagonismo entre el respeto por la promesa efectuada y el manifestado por la autonomía personal del contratante pudiere amenazar el rol fundamental de sociabilidad entre las personas, la autonomía personal continúa ocupando un lugar preponderante en el derecho contractual. ${ }^{20}$ Desde este punto de vista, el esfuerzo de Fried por articular una teoría general de la obligación contractual sobre

contrato en las prácticas contractuales cotidianas, puede hallarse en Pereira Fredes (2019b, pp. 261-306).

19 En esta línea, véase Pereira Fredes (2016, pp. 87-123). El valor de la obligación contractual desacoplándolo de la promesa está esgrimido en Molina Rodríguez (2019, pp. 167-188).

${ }^{20}$ La función de sociabilidad de las promesas está enfatizada en Arendt (2005, p. 265). 
la base del fundamento de su fuerza vinculante, puede ser replicado a partir de la autonomía personal de las partes. No es descartable que esta discusión sobre la justificación normativa del pacta sunt servanda se convierta en una estrategia de la reflexión filosófica para construir una teoría del derecho contractual. Si así fuere el caso, por ejemplo, la propuesta de Hevia debería retrotraer sus observaciones y evaluarlas según esta variante como un prometedor punto de partida. ${ }^{21}$

El problema restante en el contexto de la fundamentación del derecho de contratos radica en la indagación si acaso hay o no un único sustento normativo en esta parcela de lo jurídico. Tal observación echa luz acerca del modo de entender el derecho contractual desplegado por parte de la visión tradicional que lo entiende a partir de una pretensión de uniformidad que también permeó la filosofía del derecho de contratos. ${ }^{22}$ De manera tradicional, este ámbito jurídico ha sido interpretado de acuerdo con un canon explicativo y justificativo común y unitario. Este recae en el individualismo filosófico y su vigencia se expande a las distintas preocupaciones del fenómeno jurídico privado. ${ }^{23} \mathrm{Si}$ bien es posible hallar diversas maneras de entender el individualismo, desde un punto de vista normativo, este importa la separación entre el interés propio y el interés ajeno y, aún con mayor relevancia, el predominio del primero sobre el segundo. En el campo contractual, por ejemplo, el contratante debe privilegiar sus intereses y conducirse en aras de maximizar los réditos que le brinde el acuerdo contractual, en la medida en que no transgreda o lesione los intereses o derechos del otro contratante. ${ }^{24}$ No debe, por el contrario, actuar para favorecer o beneficiar directamente a la otra parte del contrato, resultando suficiente con que evite dañarlo. Esta forma de entender la fundamentación norma-

${ }^{21}$ Un agudo examen del rendimiento de la noción de justo precio como indicador de la estructura normativa del derecho de contratos está desarrollado en Reyes Barros, 2020, pp. 143-172.

22 Para tal ambición en la teoría jurídica analítica, véase Hart, 1963, p. 41.

23 Acerca del origen y la relevancia del individualismo en el derecho privado moderno puede consultarse Solari, 1946, pp. 3-58. Para una reconstrucción sobre las raíces intelectuales individualistas que informaron el derecho privado y su repercusión teórica, véase Pereira Fredes, 2021, pp. 139-150. El debilitamiento de la imagen individualista de este ámbito jurídico se encuentra revisado en Solari, 1950, pp. 1-10.

${ }^{24}$ En este sentido, Kennedy, 1976, p. 166. 
tiva del derecho contractual afianza la operatividad de la autonomía de la voluntad en el escenario contractual.

Desde luego, el individualismo tiene una posición si se quiere de primacía en el derecho contractual - y en el derecho privado general - pero el desafío de la filosofía del derecho de contratos reside en determinar si es correcta o no su unicidad en la estructura de fundamentación normativa que esa esfera presenta. Hay también otros posibles modelos de fundamentación que podrían hacer frente a la aparente homogeneidad del individualismo. Así, es posible pensar en el altruismo y en la solidaridad. ${ }^{25}$ Mientras que el primero en su sentido moderado pone en entredicho la prevalencia del interés personal sobre el ajeno, exige tener en permanente consideración los intereses de las demás personas y, a veces, insta a actuar derechamente a favor del interés del otro, el segundo afirma la continuidad de intereses entre las partes contratantes y, por tanto, exige actuar solidariamente a favor de la otra parte del contrato porque hay una comunión de intereses entre ellas. Como podrá apreciarse, el altruismo moderado no presupone lo que presupone la solidaridad - continuidad de intereses entre las partes contratantes- y de ahí que puede encajar en un mayor espectro de la contratación que el ideal regulativo de la solidaridad. Sencillamente, el caso central de la relación contractual es aquel en que los intereses entre las partes son discrepantes y no el supuesto en que hay comunión de aquellos. ${ }^{26}$

Una teoría del derecho de contratos hoy en día debe ofrecer una respuesta frente al interrogante sobre la fundamentación normativa del derecho de contratos y ello tiene impacto en la forma en que se articula tal teoría. Si echamos mano nuevamente a la propuesta de Hevia, es posible observar que este debate aún no era albergado al tiempo de su formulación,

25 Sobre el altruismo moderado como modelo de fundamentación normativa del derecho de contratos, véase Pereira Fredes, 2018, pp. 140-142; 2020, pp. 219-234.

${ }^{26}$ Este podría ser el caso de los contratos relacionales en los cuales, en lugar de un mero intercambio transaccional y discreto, se forja una relación entre las partes sobre la base de su interés compartido. Puede pensarse en contratos como el de sociedad, distribución y joint venture, pero evidentemente reflejan una parte del fenómeno contractual. En otro orden de cosas, Daniel Markovits ha mostrado que un prisma puramente individualista es incapaz para capturar el ideal de comunidad y respeto que se forma en la relación contractual, con base en la colaboración que media entre los contratantes en las distintas figuras contractuales. Al respecto, véase Markovits, 2004, pp. 1417-1518. 
mas de la mano de nociones como libertad, consentimiento, reciprocidad puede sospecharse que esa teoría del derecho contractual está en sintonía con una forma de individualismo desinteresado. Esto importa que los contratantes pueden actuar en función de alcanzar sus intereses contractuales, pero no pueden lesionar los de la otra parte del contrato. De ahí que nociones como la libertad y el consentimiento se encuentren en una posición privilegiada y, a su vez, la reciprocidad sea una manera de conjugar el predominio normativo del autointerés con el respeto por los intereses del otro contratante. El problema, y que no es advertido por esta posible reconstrucción, es que en el derecho de contratos también hay espacio para demandas positivas de actuación establecidas en el exclusivo interés de la otra parte del contrato. Tales deberes desafían la preeminencia del interés personal sobre el ajeno y, en su lugar, instan a actuar derechamente a favor de este último. Un camino que ha transitado paulatinamente la teoría del derecho contractual es buscar el modo de emparentar sus institutos característicos con aquel fundamento normativo que les sea más pertinente. Esto supone aceptar que no todas las instituciones responden a parámetros análogos ni están orientados en la misma dirección. Por ello, la determinación de este único sustento normativo o bien de más de uno de ellos deviene una tarea crucial.

Cuando prestamos atención a las finalidades en el derecho de contratos, estas naturalmente se ajustan a las distintas versiones de la idea de justicia; a saber, justicia correctiva, distributiva y punitiva. Hay áreas del derecho privado en que la reflexión filosófica actual acepta con menores reservas que hay una concurrencia de estas tipologías de justicia, como ocurre con el derecho de daños. ${ }^{27}$ Sin embargo, esa cuestión en el derecho contractual constituye aún un diagnóstico preliminar. ${ }^{28}$ Uno de los cargos que propinó Papayannis a Hevia estuvo asociado a la asunción de este último de que el derecho contractual es solo una cuestión de justicia correctiva. Por consiguiente, la justicia distributiva es ajena al derecho de contratos y también al derecho privado

${ }^{27}$ El enfoque de justicia distributiva en un sentido débil en el derecho de daños se encuentra defendido en Keren-Paz, 2016, en particular, 21-41. Sobre la pertinencia de las consideraciones punitivas en el derecho de daños, véase Pereira Fredes, 2015b, pp. 61-78.

${ }^{28}$ En relación con el derecho contractual y la justicia distributiva puede consultarse Kronman, 1980, pp. 472-511 y Bagchi, 2014, pp. 193-211. 
general. En su comentario crítico, Papayannis abordó diversas consideraciones para dar cuenta de la participación de demandas distributivas en el derecho de contratos, distanciándose de la tesis según la cual únicamente esta parcela de lo jurídico alberga consideraciones de justicia correctiva. En estos términos, Papayannis está afirmando que a nivel de propósitos el derecho contractual cumple, al menos, dos de manera diferenciada: responde tanto a cuestiones de justicia correctiva como distributiva. El mérito de su tesis reside, a mi juicio, en situar el problema de esta última clase de justicia en una zona que es tradicionalmente aceptada como de mayor comodidad para los asuntos de justicia correctiva. Al lograr posicionar allí la justicia distributiva podrá traspalarse con menor dificultad a otras esferas del derecho privado que son menos reticentes a las demandas distributivas. ${ }^{29}$

Este diálogo entre formas diversas de entender las demandas de la justicia abre dos caminos de análisis para la filosofía del derecho de contratos. En primer lugar, el posicionamiento de la justicia correctiva, distributiva y, si es el caso, punitiva en el derecho contractual conlleva repensar el tipo de fenómeno que es el contractual. En distintas ocasiones, la literatura pierde de vista el hecho de que la aparente presencia unitaria de la justicia correctiva en el ámbito contractual es antes una consecuencia que un presupuesto. Se trata más bien de una consecuencia toda vez que tal consideración se extrae a partir de una imagen del derecho de contratos construida en términos de uniformidad, continuidad y homogeneidad. Desde la vereda contraria, en cambio, habría que entender que, dado que el derecho contractual constituye un fenómeno caracterizado por su complejidad, diversidad y heterogeneidad, la pertinencia de más de un objetivo para esta área resulta absolutamente válida y pertinente. En la discusión entre Hevia y Papayannis se sentaron las bases para comenzar a debatir acerca del tipo de fenómeno que es el derecho de contratos - y el derecho privado-y de ahí que resulta natural prever que

29 Tal estrategia se encuentra reforzada en Papayannis, 2016a, pp. 303-368. Del mismo modo, para efectos de posicionar el altruismo moderado como fundamento normativo del derecho privado se ha acudido al derecho de contratos y derecho de propiedad, aduciendo que se trata de los ámbitos en que con mayor dificultad se acepta la presencia de directrices altruistas. En este sentido, Pereira Fredes, 2020a, pp. 261-292. Al lograr situarlas allí, sería más cómodo develar su participación en otras áreas del derecho privado como, por ejemplo, derecho de daños, derecho de familia y derecho de sucesiones. 
el ejercicio intelectual se trasladará a preguntarse por los rasgos distintivos del derecho contractual que justifican la presencia de una o más formas de justicia como finalidades a las cuales cabe aspirar.

En segundo lugar, la estrategia de hacer hincapié en las instituciones del derecho de contratos cobra especial sentido cuando se trata de tematizar el compromiso de esta parcela con una o más clases de justicia. Buena parte de las transformaciones del escenario contractual está ilustrada en la configuración de institutos que se desmarcan de la comprensión tradicional del contrato - de la cual Hevia parece comulgar en su construcción teórica-y que respecto del propósito que persiguen tampoco parecen encajar con las observaciones tradicionales de justicia correctiva. Al pensar en la teoría de la imprevisión, la ventaja injusta, la lesión subjetiva-objetiva, el abuso del derecho o la relación de consumo resulta inevitable tener en cuenta consideraciones de justicia distributiva. Un panorama por el cual la filosofía del derecho de contratos debiere encauzar sus investigaciones es a un mayor nivel de sensibilidad por el modo en que operan las instituciones más representativas de este ámbito. A partir de ellas, como fue antes sugerido, será pertinente desprender un sentido o significado sustantivo que caracterice el fenómeno contractual. Por lo demás, una alternativa para conectar la filosofía del derecho de contratos con la teoría general del contrato que está en constante revisión por los estudios dogmáticos es ofrecida precisamente gracias a las instituciones contractuales. A este entrelazamiento debiere aspirar la teoría del derecho contractual y los réditos serían significativos: atenuar la brecha que aún existe entre quienes se ocupan de la filosofía del derecho de contratos y quienes despliegan los estudios dogmáticos sobre este ámbito del fenómeno jurídico privado. Desde luego, esta brecha es más bien aparente que cierta pues ambas tareas son en verdad complementarias entre sí y persiguen la comprensión del fenómeno contractual desde énfasis distintos. Mas para la reflexión filosófica resulta indispensable desplegar sus investigaciones con una fuerte sensibilidad por el derecho de contratos que tenemos, y según las modalidades en que este efectivamente se nos presenta a diario. ${ }^{30}$

${ }^{30}$ Para una cuidada evaluación sobre el rendimiento de la reflexión teórica acerca del derecho de contratos y los estudios de dogmática puede consultarse Jiménez Castro, 2017, pp. 395-421. 


\section{Conclusiones}

El número XV de Discusiones abona el lugar de excelencia que esta publicación tiene en los estudios sobre teoría del derecho. Al prestar atención en la reflexión filosófica sobre el derecho privado y, en particular, acerca del derecho contractual devela de qué manera debe efectuarse una teoría del derecho robusta, comprometida y no estéril. Tal ejercicio intelectual vale la pena calificarlo de teoría jurídica analítica. La jurisprudencia analítica se mostró desde temprano particularmente interesada tanto por las cuestiones de explicación como por aquellas relativas a la justificación de las distintas esferas del fenómeno jurídico, exigiendo estudiarlo del modo en que este se presenta para agudizar nuestra comprensión acerca de su composición, institutos y prácticas sociales distintivas. ${ }^{31}$

Esta forma de proceder inspira el diálogo entre la teoría del derecho contractual de Hevia y sus comentaristas. Se trata de una cuestión de primera importancia forjar una teoría del derecho de contratos que capture la complejidad del fenómeno contractual. Para ello, resulta indispensable tomarse filosóficamente en serio el derecho de contratos y este número de Discusiones muestra precisamente la relevancia de aquello, ofreciendo pistas acerca de cómo llevarlo a cabo. El reto que se delinea para el programa de investigación de la filosofía del derecho contractual contemporánea es evaluar hasta qué punto en la articulación de una teoría del derecho de contratos persiste el tránsito entre el derecho y la justicia. Una empresa de esta índole debe lograr capturar ambas dimensiones para honrar la complejidad del tipo de fenómeno que persigue tanto describir como justificar. El resultado de este esfuerzo podrá ser suscrito sin reservas como una genuina teoría del derecho de contratos. ${ }^{32}$

${ }^{31}$ En relación con estos compromisos y la ausencia de una identidad unitaria y común en la tradición analítica de la filosofía del derecho, puede consultarse Pereira Fredes, 2015a, pp. 283-322; 2019a, pp. 55-104. El enriquecimiento de nuestra compresión sobre el fenómeno jurídico contractual fue también apuntado en la introducción de este número, disponible en Grosman, 2014, p. 14.

32 Una generosa gama de reflexiones acerca del derecho contractual puede encontrarse en Kraus, 2004, pp. 687-751; Lucy, 2004, pp. 75-108; y, recientemente, Markovits, D. y Emad, A., 2021. 


\section{Bibliografía}

Arendt, H. (2005). La condición humana (trad. R. Gil Novales). Barcelona: Paidós.

Atiyah, P. S. (1986a). Contract and Fair Exchange. En P. S. Atiyah, Essays on Contract (pp. 329-354). Oxford: Clarendon Press.

Atiyah, P. S. (1986b). The Modern Role of Contract Law. En P. S. Atiyah, Essays on Contract (pp. 1-9). Oxford: Clarendon Press.

Bagchi, A. (2014). Distributive Justice and Contract. En G. Klass, G. Letsas y P. Saprai, (eds.), Philosophical Foundations of Contract Law (pp. 193211). Oxford: Oxford University Press.

Barceló Compte, R. (2009). Ventaja injusta y protección de la parte débil del contrato. Madrid-Barcelona: Marcial Pons.

Benson P. (1995). The Idea of a Public Basis of Justification for Contract. Osgoode Hall Law Journal, 33(2), 273-336.

Benson P. (2019). Justice in Transactions. A Theory of Contract Law. Cambridge: The Belknap Press of Harvard University Press.

Dagan, H. y Dorfman, A. (2016). Just Relationships. Columbia Law Review, 116(6), 1395-1460.

Dagan, H. y Heller, M. (2017). The Choice Theory of Contracts. Cambridge: Cambridge University Press.

De la Maza Gazmuri, Í. (2014). Razonabilidad y responsabilidad: a propósito de la teoría del derecho contractual del profesor Martín Hevia. Discusiones, 15(2), 85-100.

Dworkin, R. (2012a). El imperio de la justicia, 2. a ed. (trad. C. Ferrari). Barcelona: Gedisa.

Dworkin, R. (2012b). Una cuestión de principios (trad. V. Boschiroli). Buenos Aires: Siglo Veintiuno Editores.

Freeman, S. (2018). Liberalism and Distributive Justice. Oxford: Oxford University Press.

Fried, C. (1996). La obligación contractual. El contrato como promesa (trad.

P. Ruíz-Tagle). Santiago de Chile: Editorial Jurídica de Chile.

Gardner, J. (2017). Dagan and Dorfman on the Value of Private Law. Columbia Law Review Online, 117, 179-201. 
Gordley, J. (1994). Myths of the French Civil Code. The American Journal of Comparative Law, 42(3), 459-505.

Grosman, L. S. (2014). Introducción: Filosofía del derecho contractual. Discusiones, 15(2), 7-14.

Hart, H. L. A. (1963). El concepto de derecho (trad. G. R. Carrió). Buenos Aires: Abeledo-Perrot.

Hevia, M. (2013). Reasonableness and Responsibility. A Theory of Contract Law. Dordrecht: Springer.

Hevia, M. (2014a). Razonabilidad y responsabilidad: Rawls, Kant y la teoría del derecho contractual. Discusiones, 15(2), 15-47.

Hevia, M. (2014b). Respuesta a las críticas. Discusiones, 15(2), 145-168.

Hevia, M. (2018). Los límites del consentimiento en el derecho contractual. En D.M. Papayannis y E. Pereira Fredes (eds.), Filosofía del derecho privado (pp. 107-118). Madrid-Barcelona: Marcial Pons.

Jiménez Castro, F. (2017). Teoría del derecho de contratos y su relación con la dogmática. Revista Chilena de Derecho, 44(2), 395-421.

Kant, I. (1999). La metafísica de las costumbres, 3. ${ }^{\text {a }}$ ed. (trad. A. Cortina Orts y J. Conill Sancho). Madrid: Tecnos.

Kennedy, D. (1976). Form and Substance in Private Law Adjudication. Harvard Law Review, 89(8), 1685-1778.

Keren-Paz, T. (2016). Derecho de daños, igualdad y justicia distributiva (trad. G. Perrotta). Madrid-Barcelona: Marcial Pons.

Kimel, D. (2018). De la promesa al contrato: Hacia una teoría liberal del contrato (trad. M. Parga). Madrid-Barcelona: Marcial Pons.

Kraus, J. S. (2004). Philosophy of Contract Law. En J.L. Coleman y S. Shapiro (eds.), The Oxford Handbook of Jurisprudence and Philosophy of Law (pp. 687-751). Oxford: Oxford University Press.

Kronman, A. T. (1980). Contract Law and Distributive Justice. The Yale Law Journal, 89(472), 472-511.

Lucy, W. (2004). Philosophy and Contract Law. University of Toronto Law Journal, 54, 75-108.

MacCormick, N. (2011). Instituciones del derecho (trad. F. Atria y S. Tschorne). Madrid-Barcelona: Marcial Pons.

Macneil, I. R. (1974). The Many Futures of Contract. Southwestern California Law Review, 47, 691-816. 
Markovits, D. (2004). Contract and Collaboration. The Yale Law Journal, $113,1417-1518$.

Markovits, D. y Emad, A. (2021). Philosophy of Contract Law. En E.N. Zalta (ed.), The Stanford Encyclopedia of Philosophy (Winter 2021 Edition), forthcoming URL $=<$ https://plato.stanford.edu/archives/ win2021/entries/contract-law/>.

Molina Rodríguez, C. (2019). El valor de la obligación contractual. En E. Pereira Fredes, (ed.), Fundamentos filosóficos del derecho civil chileno (pp. 167-188). Santiago de Chile: Rubicón Editores.

Papayannis, D. M. (2014). La insoportable levedad de la justicia correctiva en el derecho contractual. Discusiones, 15(2), 101-144.

Papayannis, D. M. (2016a). El derecho privado como cuestión pública. Bogotá: Universidad Externado de Colombia.

Papayannis, D. M. (2016b). Internismo y externismo en el derecho privado. En G. Lariguet (comp.), Metodología de la investigación jurídica: propuestas contemporáneas (pp. 439-448). Córdoba: Editorial Brujas.

Papayannis, D. M. y Pereira Fredes, E. (2018a). Estudio introductorio y análisis crítico de la teoría contractual de Dori Kimel. En D. Kimel, De la promesa al contrato: hacia una teoría liberal del contrato (pp. 13-50). Madrid-Barcelona: Marcial Pons.

Papayannis, D. M. y Pereira Fredes, E. (2018b). Introducción: sobre la filosofía del derecho privado. En D.M. Papayannis y E. Pereira Fredes (eds.), Filosofía del derecho privado (pp. 15-42). Madrid-Barcelona: Marcial Pons.

Pereira Fredes, E. (2015a). Tradición analítica de la filosofía del derecho. En F. Vergara Ceballos (ed.), Problemas actuales de la filosofía jurídica (pp. 283-322). Santiago de Chile: Librotecnia.

Pereira Fredes, E. (2015b). Un alegato a favor de las consideraciones punitivas en el derecho privado. Revista de Derecho. Escuela de Postgrado, 7, 61-78.

Pereira Fredes, E. (2016). ¿Por qué obligan los contratos? Justificación normativa de la obligatoriedad del vínculo contractual. Santiago de Chile: Thomson Reuters.

Pereira Fredes, E. (2017). ¿Filosofía del derecho privado? En J.C. Marín González y A. Schopf Olea (eds.), Lo público y lo privado en el derecho. 
Estudios en homenaje al profesor Enrique Barros Bourie (pp. 193-261). Santiago de Chile: Thomson Reuters.

Pereira Fredes, E. (2018). Altruismo y derecho contractual. En D.M. Papayannis y E. Pereira Fredes (eds.), Filosofía del derecho privado (pp. 139-168). Madrid-Barcelona: Marcial Pons.

Pereira Fredes, E. (2019a). Carrió y la filosofía analítica del derecho. En D. Sierra Sorockinas (ed.), Notas (al margen) sobre Derecho y Lenguaje (pp. 55-104). Bogotá: Universidad Externado de Colombia.

Pereira Fredes, E. (2019b). Muerte del contrato. En E. Pereira Fredes (ed.), Fundamentos filosóficos del derecho civil chileno (pp. 261-306). Santiago de Chile: Rubicón Editores.

Pereira Fredes, E. (2020a). Algunas maneras de mostrar el altruismo en el derecho privado. En J.A. García Amado y D.M. Papayannis (eds.), Dañar, incumplir y reparar. Ensayos de filosofía del derecho privado (pp. 217-299). Lima: Palestra.

Pereira Fredes, E. (2020b). La buena fe y sus fundamentos normativos. Latin American Legal Studies, 7, 111-142.

Pereira Fredes, E. (2021). ¿Cómo repensar la pobreza desde el derecho privado? En C. Fernández Blanco y E. Pereira Fredes (eds.), Derecho y pobreza (pp. 129-170). Madrid-Barcelona: Marcial Pons.

Rawls, J. (1995a). Liberalismo político (trad. S. R. Madero Báez). Ciudad de México: Fondo de Cultura Económica.

Rawls, J. (1995b). Teoría de la justicia, 2. a ed. (trad. M. D. González). Ciudad de México: Fondo de Cultura Económica.

Rawls, J. (2004). La justicia como equidad. Una reformulación (trad. A. de Francisco). Buenos Aires: Paidós.

Rengifo Gardeazabal, M. (2014). Teoría del contrato, libertad y determinismo social: la posición incompatibilista. Discusiones, 15(2), 49-84.

Reyes Barros, J. (2020). Más allá de la justicia conmutativa: Derecho de contratos, justicia y precios justos. Latin American Legal Studies, 7, 143-172.

Scheffler, S. (2015). Distributive Justice, the Basic Structure and the Place of Private Law. Oxford Journal of Legal Studies, 35(2), pp. 213-235. 
Scheinman, H. (2000). Contractual Liability and Voluntary Undertakings. Oxford Journal of Legal Studies, 20(2), 205-220.

Smith, S. A. (2004). Contract Theory. Oxford: Oxford University Press.

Solari, G. (1946). Filosofía del derecho privado. I. La idea individual (trad. O. Caletti). Buenos Aires: De Palma.

Solari, G. (1950). Filosofía del derecho privado. II. La idea social (trad. O. Caletti). Buenos Aires: De Palma.

Tarello, G. (2002). Ideologías del siglo XVIII sobre la codificación y estructura de los códigos. En G. Tarello, Cultura jurídica y política del derecho (pp. 43-64) (trad. J. L. Monereo Pérez). Granada: Comares.

Weinrib, E. J. (2012). Corrective Justice. Oxford: Oxford University Press.

Weinrib, E. J. (2017). La idea de derecho privado (trad. E. Paez). MadridBarcelona: Marcial Pons. 